\title{
Modélisation de l'efficacité de la prévention pour estimer le point de bascule de l'équité : quelle couverture des interventions préventives individuelles permet de réduire les effets des disparités socioéconomiques relatives au risque de diabète?
}

D. G. Manuel, M. Sc., M.D. (1, 2, 3, 4, 5); T. H. Ho, M. Sc. (1); S. Harper, Ph. D. (6); G. M. Anderson, M.D., Ph. D. (1, 7); J. Lynch, Ph. D. (8, 9); L. C. Rosella, Ph. D. $(1,2,10)$

Cet article a fait l'objet d'une évaluation par les pairs.

\section{Résumé}

Introduction : La plupart des traitements préventifs individuels peuvent atténuer ou renforcer les disparités en santé selon leur efficacité différentielle dans la collectivité en fonction du statut socioéconomique (SSE). Le point de bascule de l'équité — défini comme le point à partir duquel les disparités en santé augmentent - se calcule en faisant varier les composantes de l'efficacité dans la collectivité, par exemple le risque de base de la maladie, la couverture des interventions ou l'efficacité de ces dernières, en fonction du SSE.

Méthodologie : Nous avons utilisé une méthode simple de modélisation pour estimer l'efficacité de la prévention du diabète dans la collectivité au Canada selon le SSE selon divers scénarios de couverture d'intervention.

Résultats : Le risque de base de diabète à cinq ans variait de 1,76\% entre le groupe ayant le revenu le plus faible et celui ayant le revenu le plus élevé. Lorsqu’on supposait que la couverture était complète dans toutes les tranches de revenu, l'écart diminuait, passant à 0,90\% (prévention de 144000 cas) à la suite d'interventions sur le mode de vie et à 1,24\% (prévention de 88100 cas) au moyen de la pharmacothérapie. Le point de bascule de l'équité a été estimé comme étant un écart de couverture de $30 \%$ dans le cas des interventions de prévention (100 \% de couverture dans le groupe ayant le revenu le plus élevé et $70 \%$ de couverture dans le groupe ayant le revenu le plus faible).

Conclusion : Les disparités relativement au risque de diabète pourraient être sensiblement réduites si les interventions étaient adoptées de manière égale dans tous les groupes indépendamment du SSE. Cependant, les disparités en matière de couverture sont susceptibles d'entraîner une plus grande inégalité du risque. Des méthodes simples de modélisation peuvent servir à déterminer l'efficacité des interventions de prévention individuelles dans la collectivité et leur potentiel à réduire (ou augmenter) les disparités. Le point de bascule de l'équité peut être utilisé comme seuil critique dans l'analyse des disparités.

Mots-clés : inégalités, santé publique, évaluation des incidences sur la santé, diabète

\section{Introduction}

Le rôle du traitement préventif individuel dans la réduction des disparités en matière de santé est actuellement l'objet de débats. D’une part, ces disparités peuvent être atténuées lorsque les effets relatifs d'un traitement sont constants dans le spectre des risques sous-jacents ${ }^{1-3}$. Si cette hypothèse se vérifiait, l'efficacité absolue des interventions en santé dans la collectivité serait plus importante dans les groupes défavorisés que dans les autres groupes, car les groupes défavorisés présentent généralement un risque de base plus élevé. Par conséquent, si les personnes dont le statut socioéconomique (SSE) est le plus bas réalisaient des gains en bénéfices absolus plus importants que celles dont le SSE est le plus élevé, alors les disparités qui existent entre elles s'amenuiseraient ${ }^{1}$.

D'autre part, on ne peut présumer de la constance de l'efficacité des interventions, et, par ailleurs, la couverture des interventions est souvent inéquitable. La couverture d'une intervention, soit la proportion de la population cible qui adopte cette intervention, dépend des facteurs ayant une incidence sur l'adoption de cette intervention comme l'exactitude avec

Rattachement des auteurs :

1. Institut de recherche en services de santé, Toronto (Ontario), Canada

2. École de santé publique Dalla Lana, Université de Toronto, Toronto (Ontario), Canada

3. Institut de recherche de l'Hôpital d'Ottawa, Ottawa (Ontario), Canada

4. Statistique Canada, Ottawa (Ontario), Canada

5. Département de médecine familiale et Département d'épidémiologie et de médecine communautaire, Université d'Ottawa, Ottawa (Ontario), Canada

6. Département d'épidémiologie, de biostatistique et de santé au travail, Université McGill, Montréal, (Québec), Canada

7. Département de gestion et d'évaluation des politiques de santé, Université de Toronto, Toronto, (Ontario), Canada

8. School of Population Health and Clinical Sciences, Université d'Adelaide, Adelaide, Australie

9. Department of Social and Community Medicine, Université de Bristol, Bristol, Royaume-Uni

10. Santé publique Ontario, Toronto (Ontario), Canada

Correspondance : Douglas G. Manuel, Institut de recherche en santé d’Ottawa, immeuble des services administratifs 1-008, 1053, avenue Carling, Ottawa (Ontario) K1Y 4E9; tél. : 613-798-5555, poste 19108; téléc. : 613-761-5492; courriel : dmanuel@ohri.ca 
laquelle on a déterminé la population cible, le taux de participation à l'intervention et l'adhésion des consommateurs ${ }^{4}$. Les groupes à statut socioéconomique faible rencontrent souvent des obstacles dans l'accès aux soins, du fait par exemple d'effets liés au revenu, ou adhérent moins aux traitements préventifs, en raison par exemple d'effets liés au niveau de scolarité $^{5}$. Ensemble, ces écarts d'efficacité et de couverture compromettent les efforts déployés pour réduire les disparités et ils peuvent même conduire, paradoxalement, à les augmenter ${ }^{6}$.

Dans notre étude, nous montrons comment une méthode simple de modélisation peut être utilisée pour déterminer dans quelle mesure les interventions de prévention permettent de réduire les disparités (voir la formule 1). Pour ce faire, nous avons choisi comme sujet la prévention du diabète au Canada.

\section{FORMULE 1}

\section{Efficacité dans la communauté = population cible $\times$ risque de base $\times$ efficacité de l'intervention $x$ couverture de l'intervention*}

Efficacité dans la collectivité : bénéfice (ou réduction absolue du risque) associé aux interventions préventives dans un milieu communautaire réel.

Population cible : proportion de la population (ou chaque strate sociodémographique) admissible à l'intervention préventive.

Risque de base : risque ou probabilité de développer une maladie avant la mise en œuvre de l'intervention.

Efficacité de l'intervention : bénéfice relatif d'une intervention déterminé dans les essais d'intervention ou d'autres études, c.-à-d. dans un milieu idéal ou contrôlé.

Couverture de l'intervention : proportion de la population cible qui adopte l'intervention.

*Inspiré de la " boucle efficacité-équité »".
Nous pouvons estimer le potentiel des interventions à réduire les inégalités en calculant et comparant leur efficacité dans la collectivité pour chacune des strates socioéconomiques. Les disparités liées à la maladie vont s'amenuiser si l'efficacité est plus grande dans les groupes à faible SSE que dans les groupes à SSE élevé.

L'une des difficultés fréquemment rencontrées lorsqu'il est question d'estimer l'efficacité dans la collectivité pour chacune des strates socioéconomiques est le manque d'information propre aux différentes strates, notamment les variations de taille de la population cible, le risque de base, l'efficacité de l'intervention et sa couverture. Pour rendre compte de ces situations, nous avons introduit le concept de « point de bascule de l'équité ", défini comme le point à partir duquel la disparité entre les strates socioéconomiques basse et élevée augmente en raison de différences dans l'efficacité des interventions, la couverture ou la population cible. L'objectif du calcul du point de bascule de l'équité est d'évaluer si les différences potentielles existent réellement. Dans l'exemple de la prévention du diabète, nous cherchions à établir le point à partir duquel la couverture insuffisante des interventions menait à une plus grande disparité relativement au risque de diabète. Pour mieux illustrer cela, nous n'avons pas fait varier l'efficacité des interventions ni les critères de la population cible.

Nous avons examiné deux types d'interventions de prévention du diabète dont l'efficacité est prouvée : les interventions sur le mode de vie et la pharmacothérapie ${ }^{7}$. Les interventions sur le mode de vie diminuent l'obésité par l'alimentation et des programmes d'exercices. La pharmacothérapie fait appel à des médicaments comme la metformine pour perdre du poids et réduire la résistance à l'insuline, dans le but de diminuer le nombre de nouveaux cas de diabète chez les personnes à risque. Quoique ces traitements semblent efficaces dans tous les groupes socioéconomiques ${ }^{7,8}$, il est difficile d'obtenir une couverture élevée pour le diabète, en particulier et pour les maladies chroniques en général ${ }^{9,10}$, et le degré de couverture a tendance à varier d'un groupe socioéconomique à l'autre. À notre connais- sance, il n'existe cependant aucune donnée sur la couverture des interventions en prévention du diabète en fonction du SSE.

Nous avons estimé le point de bascule de l'équité en deux étapes. D’abord, nous avons calculé l'efficacité des interventions sur le mode de vie et de la pharmacothérapie dans la collectivité dans le cadre d'un scénario où la couverture de tous les groupes socioéconomiques était de $100 \%$. Ce calcul a permis d'estimer la réduction maximale ou optimale de la disparité en matière de diabète. Par la suite, nous avons réduit progressivement la couverture dans le groupe au SSE le plus faible jusqu'à atteindre le point de bascule de l'équité, ou point à partir duquel on n'a constaté aucune réduction des disparités en matière de diabète.

\section{Méthodologie}

L'étude a été approuvée par le comité d'éthique de la recherche du Sunnybrook Health Sciences Centre de Toronto (Ontario). La figure 1 illustre les étapes suivies pour sélectionner une population cible admissible et pour évaluer l'incidence des interventions en santé sur les disparités socioéconomiques ayant des effets sur le risque de diabète.

\section{Sources des données et sélection de la cohorte}

Nous avons utilisé les données de l'Enquête sur la santé dans les collectivités canadiennes (ESCC) de 2005 pour obtenir le statut des Canadiens à l'égard du diabète, leur SSE et d'autres facteurs de risque liés au diabète ( $\mathrm{n}=107212$, non pondéré). Cette enquête est une enquête transversale en population générale représentative à l'échelle nationale et dans laquelle les données sur l'état de santé et les déterminants de la santé sont autodéclarées ${ }^{11}$. Nous avons sélectionné tous les Canadiens adultes non atteints de diabète de l'ESCC à l'exception des femmes enceintes.

\section{Population cible}

Nous avons défini la population cible par les interventions préventives à l'aide de 
FIGURE 1

Processus pour estimer l'efficacité dans la collectivité et le point de bascule de l'équité des interventions de prévention du diabète (modification du mode de vie ou pharmacothérapie). Exemple d'une réduction des disparités dans toutes les tranches de revenu à l'aide d'une modification du mode de vie

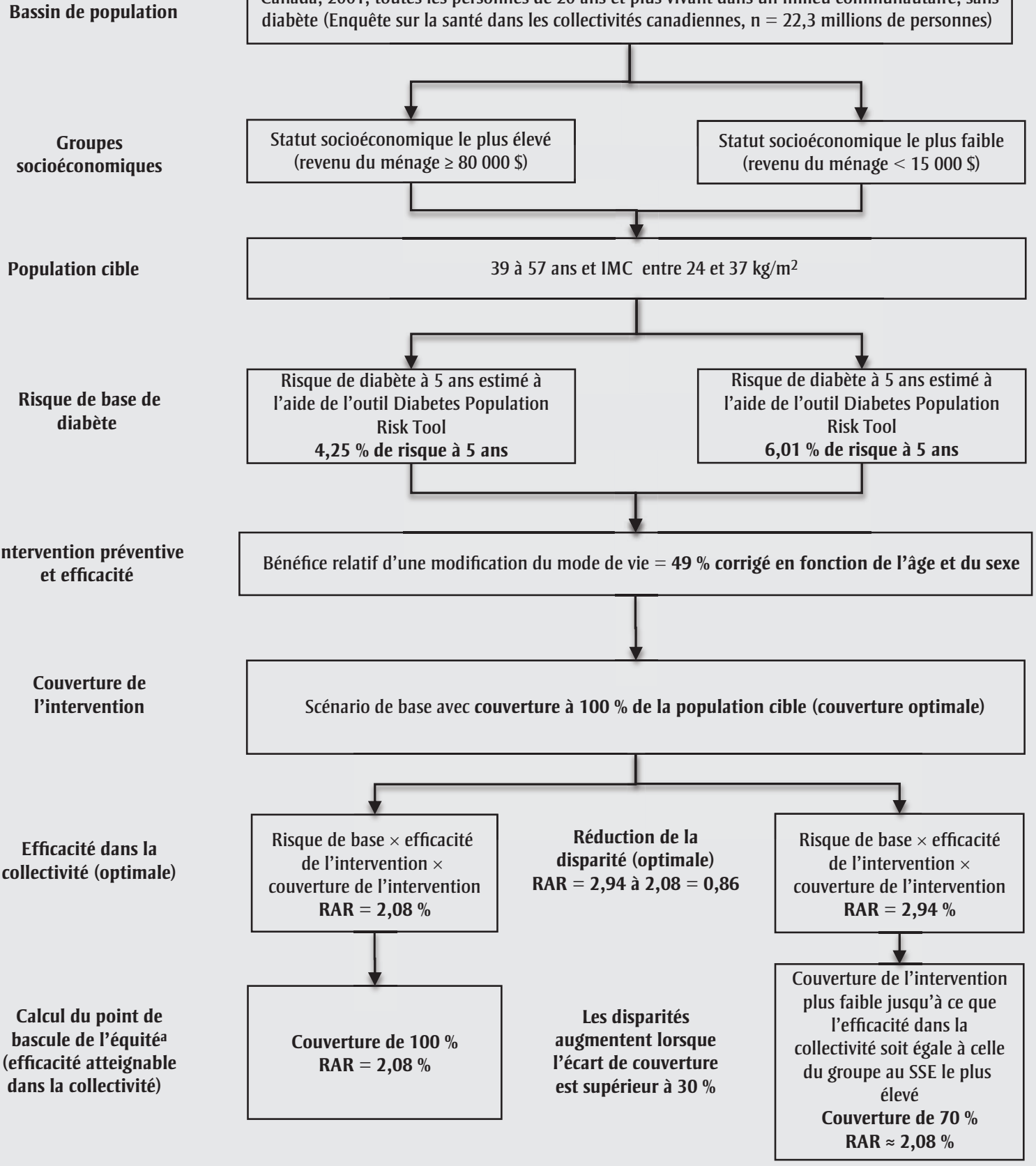

Abréviations : IMC, indice de masse corporelle; RAR, réduction absolue du rique.

${ }^{a}$ Réduction de la couverture (ou du risque de base, ou de l'efficacité ou de la population cible) lorsqu'on observe la même efficacité dans tous les groupes.

données tirées d'une méta-analyse portant sur 17 études d'efficacité de traitements qui préviennent ou retardent l'apparition du diabète chez les adultes ${ }^{7}$. Pour tenir compte de la fourchette d'âge moyen et de l'indice de masse corporelle (IMC) des personnes ayant participé à ces 17 études, nous avons défini comme population cible les personnes de 39 à 57 ans dont l'IMC se situait entre 24 et $37 \mathrm{~kg} / \mathrm{m}^{2}$. Les études examinées dans le cadre de la méta-analyse indiquaient une efficacité identique dans un large éventail de populations et de paramètres socioéconomiques.

\section{Risque de base de diabète}

Le risque de base de diabète à cinq ans (2005 à 2010) en fonction du SSE a été calculé à l'aide du Diabetes Population Risk Tool (DPoRT), un algorithme validé permettant de prédire le risque ${ }^{12}$. Nous 
avons ensuite évalué l'exactitude prédictive du DPoRT et avons établi que l'incidence du diabète peut être prédite avec discrimination et exactitude dans tous les groupes socioéconomiques (voir annexe en ligne).

\section{Efficacité des interventions préventives}

La méta-analyse portant sur les interventions faisait état d'un rapport des risques instantanés (RRI) regroupé à 5 ans de 0,51 (IC à $95 \%: 0,44$ à 0,60) dans le cas des interventions sur le mode de vie et de 0,70 (IC à $95 \%$ : 0,62 à 0,79) dans le cas des interventions médicamenteuses, ce qui donne des effets relatifs du traitement de $49 \%$ et de $30 \%$, respectivement. Au cours de la méta-analyse, les chercheurs n’ont pas évalué si l'efficacité variait en fonction du SSE.

\section{Analyses sur l'efficacité dans la collectivité}

Au moyen du revenu du ménage et du niveau de scolarité des répondants comme indicateurs du SSE, nous avons calculé le risque de diabète (et la réduction absolue du risque [RAR]) selon le SSE au début de l'étude et dans le cadre de scénarios post-intervention supposant une pleine couverture. La RAR a été définie comme la différence absolue entre le risque de base de diabète et le risque post-intervention. Par disparité, nous entendions la différence absolue du risque de diabète entre le groupe au SSE le plus faible et celui au SSE le plus élevé. Par la suite, nous avons comparé la disparité de base et la disparité postintervention pour calculer la réduction absolue. Enfin, nous avons examiné dans quelle mesure une couverture sous-optimale des interventions influait sur la disparité. Pour faciliter l'interprétation, nous avons exploré ce scénario en maintenant une couverture large dans les groupes au SSE le plus élevé et en faisant varier la couverture dans les groupes au SSE le plus faible. Nous avons calculé le point de bascule de l'équité en recalculant la RAR des groupes au SSE le plus faible à mesure que la couverture variait, jusqu'à ce que nous atteignions la même RAR que celui des groupes au SSE le plus élevé.

\section{Résultats}

Dans le tableau 1, nous avons comparé les caractéristiques des adultes du groupe cible et du celles du groupe non ciblé au début de l'étude. La population cible était principalement composée d'hommes, et par définition - comprenait surtout des adultes en surpoids ou obèses. La population cible représentait $32,8 \%$ des
TABLEAU 1

Caractéristiques démographiques, socioéconomiques et anthropométriques de référence des adultes non atteints de diabète, 20 ans et plus, Enquête sur la santé dans les collectivités canadiennes, $2005^{\mathrm{a}}$

\begin{tabular}{|c|c|c|}
\hline Caractéristiques & $\begin{array}{c}\text { Population cible }^{\mathrm{b}} \\
(\mathrm{N}=7 \mathbf{1 0 0} 000) \\
\%\end{array}$ & $\begin{array}{l}\text { Population non ciblée } \\
\qquad \begin{array}{c}\text { (N = } 15200000) \\
\%\end{array}\end{array}$ \\
\hline \multicolumn{3}{|l|}{ Sexe } \\
\hline Hommes & 60,1 & 43,4 \\
\hline Femmes & 40,0 & 56,6 \\
\hline \multicolumn{3}{|l|}{ Âge (ans) } \\
\hline 20 à 34 & - & 41,5 \\
\hline 35 à 44 & 41,5 & 13,1 \\
\hline 45 à 54 & 41,1 & 10,7 \\
\hline 55 à 64 & 17,4 & 12,8 \\
\hline 65 et plus & - & 21,9 \\
\hline \multicolumn{3}{|l|}{ Revenu du ménage (\$) } \\
\hline Moins de 15000 & 3,5 & 6,4 \\
\hline 15000 à 29999 & 7,1 & 15,1 \\
\hline 30000 à 49999 & 16,8 & 21,6 \\
\hline 50000 à 79999 & 29,1 & 26,3 \\
\hline 80000 ou plus & 43,4 & 30,6 \\
\hline \multicolumn{3}{|l|}{ Niveau de scolarité } \\
\hline Sans diplôme d'études secondaires & 12,2 & 17,1 \\
\hline Diplôme d'études secondaires & 16,3 & 15,8 \\
\hline Études postsecondaires non terminées & 6,8 & 9,2 \\
\hline Diplôme d'études postsecondaires & 64,7 & 57,9 \\
\hline \multicolumn{3}{|l|}{ Travail } \\
\hline Oui & 88,1 & 74,8 \\
\hline Non & 11,9 & 25,2 \\
\hline \multicolumn{3}{|l|}{ Groupe ethnique } \\
\hline Non de race blanche & 13,8 & 17,3 \\
\hline De race blanche & 86,2 & 82,7 \\
\hline \multicolumn{3}{|l|}{ Immigrant } \\
\hline Oui & 20,8 & 22,8 \\
\hline Non & 79,2 & 77,2 \\
\hline \multicolumn{3}{|l|}{ IMC $\left(\mathrm{kg} / \mathrm{m}^{2}\right)$} \\
\hline Poids insuffisant $(<18,50)$ & - & 3,9 \\
\hline Poids normal $(18,50$ à 24,99$)$ & 20,6 & 61,1 \\
\hline Surpoids (25,00 à 29,99) & 57,2 & 23,6 \\
\hline Obésité ( $\geq 30$ ) & 22,2 & 11,4 \\
\hline
\end{tabular}

Abréviation : IMC, indice de masse corporelle.

a Résultats basés sur des estimations pondérées tenant compte de la conception de l'enquête et des caractéristiques démographiques de la population.

${ }^{\text {b }}$ Adultes de 39 à 57 ans dont l'IMC se situe entre 24,00 et 37,00 kg/ $\mathrm{m}^{2}$. 
22,3 millions de Canadiens adultes non atteints de diabète.

En 2001, le risque de diabète de base à 5 ans au Canada était de 4,78\%. Le risque de diabète baissait quand le SSE augmentait, passant de 6,01 \% chez les personnes ayant le revenu le plus faible à 4,25\% chez celles ayant le revenu le plus élevé, d'où une disparité de base de 1,76\% entre le groupe à plus faible SSE et celui à SSE plus élevé. Le tableau 2 détaille le risque prédit de diabète en fonction des strates socioéconomiques, de l'IMC et d'autres facteurs démographiques.

Le tableau 3 illustre l'incidence d'une pleine couverture des interventions sur le mode de vie ou des interventions pharmacologiques sur les disparités en matière de risque de diabète selon le SSE. La RAR associée à une intervention sur le mode de vie était de 2,34\%. La disparité entre les personnes dont le revenu est le plus faible et celles ayant le revenu le plus élevé s'en trouvait réduite, passant ainsi de 1,76\% à $0,90 \%$. Avec la pharmacothérapie, la RAR s'élevait à $1,43 \%$, ce qui faisait passer la disparité de $1,76 \%$ à 1,24\%. Cela avait des bénéfices aussi quant au nombre total de cas de diabète prévenus au Canada (144 000 cas prévenus à l'aide d'interventions sur le mode de vie contre 88100 cas prévenus par la pharmacothérapie). Le nombre de cas de diabète prévenus dans les strates socioéconomiques basses était plus faible que dans les strates socioéconomiques élevées, signe que les strates socioéconomiques basses comportaient un plus petit nombre de personnes (voir les tableaux 1 et 2).

La figure 2 illustre l'incidence d'une pleine couverture des interventions en santé sur les disparités en matière de risque de diabète selon le revenu et le niveau de scolarité. Comme prévu, la disparité augmente lorsque la couverture des interventions chute dans les groupes au SSE le plus faible mais demeure à $100 \%$ dans les groupes au SSE le plus élevé. Le point de bascule de l'équité est atteint avec une couverture de $70 \%$ chez les personnes les moins nanties et une couverture de $60 \%$ dans les groupes ayant le niveau de scolarité le plus faible. Nous avons constaté une élévation progressive du risque de diabète entre les groupes aux

TABLEAU 2

Risque de base à cinq ans et nombre prédit de nouveaux cas de diabète dans la population cible, 2005 à 2010

\begin{tabular}{|c|c|c|}
\hline \multirow[t]{2}{*}{ Caractéristique } & \multicolumn{2}{|c|}{ Population cible $^{\mathrm{a}}$} \\
\hline & Nombre de cas ${ }^{b}$ (n) & Risque moyen $(\%)$ \\
\hline Total & 323000 & 4,78 \\
\hline \multicolumn{3}{|l|}{ Sexe } \\
\hline Hommes & 193000 & 4,77 \\
\hline Femmes & 130000 & 4,80 \\
\hline \multicolumn{3}{|l|}{ Âge (ans) } \\
\hline 20 à 34 & - & - \\
\hline 35 à 44 & 63500 & 2,26 \\
\hline 45 à 54 & 181000 & 6,40 \\
\hline 55 à 64 & 79000 & 6,63 \\
\hline 65 et plus & - & - \\
\hline \multicolumn{3}{|l|}{ Revenu du ménage (\$) } \\
\hline Moins de 15000 & 11800 & 6,01 \\
\hline 15000 à 29999 & 24700 & 5,55 \\
\hline 30000 à 49999 & 53800 & 5,05 \\
\hline 50000 à 79999 & 85700 & 4,59 \\
\hline 80000 ou plus & 118000 & 4,25 \\
\hline \multicolumn{3}{|l|}{ Niveau de scolarité } \\
\hline Sans diplôme d'études secondaires & 55300 & 6,82 \\
\hline Diplôme d'études secondaires & 62300 & 5,60 \\
\hline Études postsecondaires non terminées & 22000 & 4,53 \\
\hline Diplôme d'études postsecondaires & 184000 & 4,10 \\
\hline \multicolumn{3}{|l|}{ Travail } \\
\hline Oui & 272000 & 4,54 \\
\hline Non & 48800 & 6,21 \\
\hline \multicolumn{3}{|l|}{ Groupe ethnique } \\
\hline Non de race blanche & 68900 & 7,39 \\
\hline De race blanche & 255000 & 4,50 \\
\hline \multicolumn{3}{|l|}{ Immigrant } \\
\hline Oui & 87100 & 5,87 \\
\hline Non & 236000 & 4,63 \\
\hline \multicolumn{3}{|l|}{ IMC $\left(\mathrm{kg} / \mathrm{m}^{2}\right)$} \\
\hline Poids insuffisant $(<18,50)$ & - & - \\
\hline Poids normal $(18,50$ à 24,99$)$ & 30500 & 2,05 \\
\hline Surpoids (25,00 à 29,99) & 153000 & 3,81 \\
\hline Obésité ( $\geq 30$ ) & 140000 & 9,19 \\
\hline
\end{tabular}

${ }^{a}$ Adultes de 39 à 57 ans dont l'IMC se situe entre 24,00 et $37,00 \mathrm{~kg} / \mathrm{m}^{2}$.

b Selon des estimations pondérées tenant compte de la conception de l'enquête et des caractéristiques démographiques de la population.

valeurs inférieures à ces pourcentages de couverture.

\section{Analyse}

Notre étude présente une méthode de modélisation simple permettant d'estimer la réduction des disparités en matière de risque de maladie selon le statut socioéconomique à l'aide d'interventions de prévention individuelles. Dans le vaste débat consistant à se demander si les interventions individuelles peuvent ou devraient jouer un rôle dans la réduction 
TABLEAU 3

Incidence d'une couverture optimale (100\%) des interventions sur le mode de vie et des interventions pharmacologiques sur la disparité relativement au risque de diabète

\begin{tabular}{|c|c|c|c|c|c|c|c|c|}
\hline Revenu du ménage (\$) & $\begin{array}{c}\text { Moins de } \\
15000\end{array}$ & $\begin{array}{c}15000 \text { à } \\
29999\end{array}$ & $\begin{array}{c}30000 \text { à } \\
49999\end{array}$ & $\begin{array}{c}50000 \text { à } \\
79999\end{array}$ & $\begin{array}{l}80000 \\
\text { et plus }\end{array}$ & Total & Disparitéa $^{a}$ & $\begin{array}{c}\text { Réduction } \\
\text { absolue de la } \\
\text { disparitéb }^{\text {dis }}\end{array}$ \\
\hline Risque de base de diabète (\%) & 6,01 & 5,55 & 5,05 & 4,59 & 4,25 & 4,78 & 1,76 & - \\
\hline \multicolumn{9}{|l|}{ Intervention sur le mode de vie } \\
\hline Réduction absolue du risque ${ }^{c}(\%)$ & 2,94 & 2,72 & 2,47 & 2,25 & 2,08 & 2,34 & \multirow[t]{2}{*}{0,90} & \multirow[t]{2}{*}{0,86} \\
\hline Cas prévenus $^{\mathrm{d}}(\mathrm{n})$ & 5760 & 12100 & 26400 & 42000 & 57600 & 144000 & & \\
\hline Réduction absolue du risque $^{c}(\%)$ & 1,80 & 1,67 & 1,52 & 1,38 & 1,28 & 1,43 & \multirow[t]{2}{*}{1,24} & \multirow[t]{2}{*}{0,52} \\
\hline Cas prévenus $^{\mathrm{d}}$ (n) & 3530 & 7420 & 16100 & 25700 & 35300 & 88100 & & \\
\hline Niveau de scolarité & $\begin{array}{c}\text { Sans diplôme } \\
\text { d'études } \\
\text { secondaires }\end{array}$ & $\begin{array}{c}\text { Diplôme } \\
\text { d'études } \\
\text { secondaires }\end{array}$ & $\begin{array}{l}\text { Études post- } \\
\text { secondaires non } \\
\text { terminées }\end{array}$ & \multicolumn{2}{|c|}{$\begin{array}{l}\text { Diplôme } \\
\text { d'études post- } \\
\text { secondaires }\end{array}$} & Total & Disparitéa $^{a}$ & $\begin{array}{c}\text { Réduction } \\
\text { absolue de la } \\
\text { disparitéb }^{\text {b }}\end{array}$ \\
\hline \multicolumn{9}{|l|}{ Intervention sur le mode de vie } \\
\hline Cas prévenus $^{\mathrm{d}}(\mathrm{n})$ & 27100 & 30500 & 10800 & & & 159000 & 1,39 & 1,33 \\
\hline \multicolumn{9}{|l|}{ Intervention pharmacologique } \\
\hline Réduction absolue du risque ${ }^{c}(\%)$ & 2,05 & 1,68 & 1,36 & & & 1,43 & \multirow[t]{2}{*}{1,90} & \multirow[t]{2}{*}{0,82} \\
\hline Cas prévenus $^{\mathrm{d}}(\mathrm{n})$ & 16600 & 18700 & 6610 & & & 97000 & & \\
\hline
\end{tabular}

${ }^{\text {a }}$ Disparité = risque de diabète dans le groupe socioéconomique le plus bas - risque de diabète dans celui au statut le plus élevé

b Réduction absolue de la disparité = disparité de base - disparité post-intervention

c Réduction absolue du risque $=$ risque de base de diabète - risque de diabète post-intervention

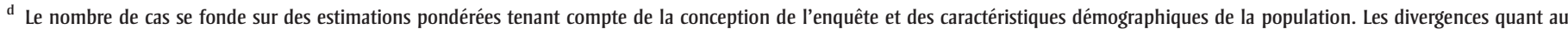
nombre total de cas prévenus sont attribuables à des données manquantes sur le revenu ou le niveau de scolarité.

des disparités en santé, la discussion est généralement étayée par des arguments théoriques ou des exemples provenant d'études ou de populations particulières ${ }^{13,14}$. En pratique, la plupart des interventions préventives - qu'elles soient individuelles ou à l'échelle de la collectivité - peuvent soit diminuer soit renforcer les disparités en santé selon la différence de risque de base, l'efficacité de l'intervention et la couverture des interventions. Il est donc utile, pour réaliser une planification pratique des services de santé et pour susciter des discussions plus générales sur la prévention des maladies, de pouvoir analyser dans quels contextes les interventions préventives vont sans doute atténuer les disparités en santé et dans quels contextes elles risquent de les accentuer.

Rose $^{15}$ a indiqué, à juste titre, que la compréhension des mesures absolues du risque est la pierre angulaire de la planification en matière de santé des populations. Cependant, les limites de la méthode de Rose pour évaluer les questions d'équité ont fait l'objet de beaucoup de débats ${ }^{6,13,16}$. Tugwell et collab. ${ }^{4}$ ont présenté un cadre permettant de prendre en compte les questions d'équité lors de l'évaluation des politiques de santé. La méthode de modélisation utilisée ici est semblable à la " boucle efficacité-équité ", décrite par Tugwell et collab. ${ }^{4}$, sauf que nos calculs étaient spécifiques aux interventions préventives et faisaient appel à un outil d'analyse multivariée du risque pour estimer le risque de base. Le point de bascule de l'équité est aussi analogue, comme mesure, aux analyses de seuil ou de sensibilité des études économiques dans lesquelles il existe une incertitude ou une variation dans l'établissement des coûts des composantes et dans les estimations de l'efficacité ${ }^{17,18}$.
Nous n'avons fait varier qu'une composante de l'efficacité dans la collectivité la couverture - pour examiner son effet sur le point de bascule de l'équité. Nous avons choisi cette variable en raison des préoccupations liées la grande variabilité de la couverture selon le groupe socioéconomique et en raison de la faible couverture des traitements préventifs du diabète observée dans les collectivités à faible revenu ${ }^{19}$. L'adoption des interventions est influencée par un ensemble complexe de facteurs liés à la personne, au fournisseur de soins et au système de santé - attitudes, barrières linguistiques et culturelles, confiance envers le système de santé, qualité de la communication et continuité des soins ${ }^{9,10,20-22}$ qui peuvent tous avoir divers effets sur la couverture des interventions.

Comme nous nous y attendions, la couverture des interventions diminue chez les 
FIGURE 2

Estimation de l'incidence de la couverture des interventions sur la disparité relativement au risque de diabète et détermination du point de bascule de l'équité dans les interventions sur le mode de vie et les interventions pharmacologiques

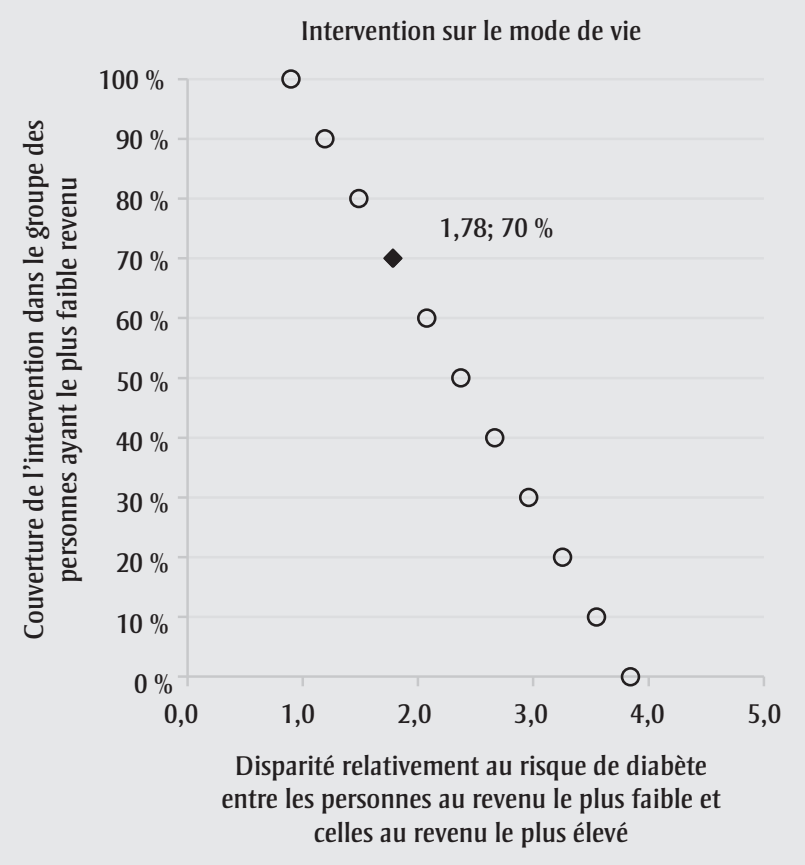

Intervention sur le mode de vie

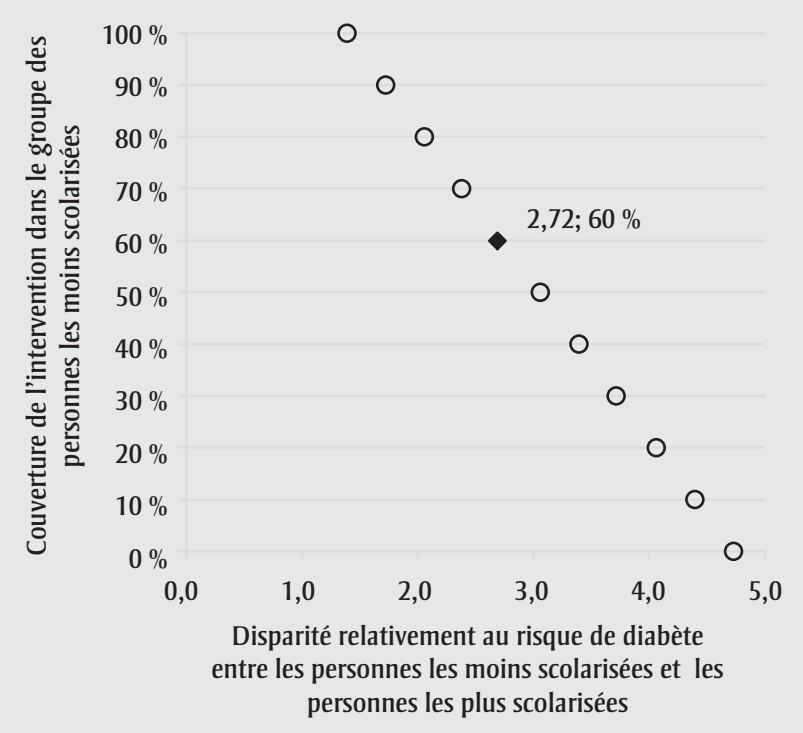

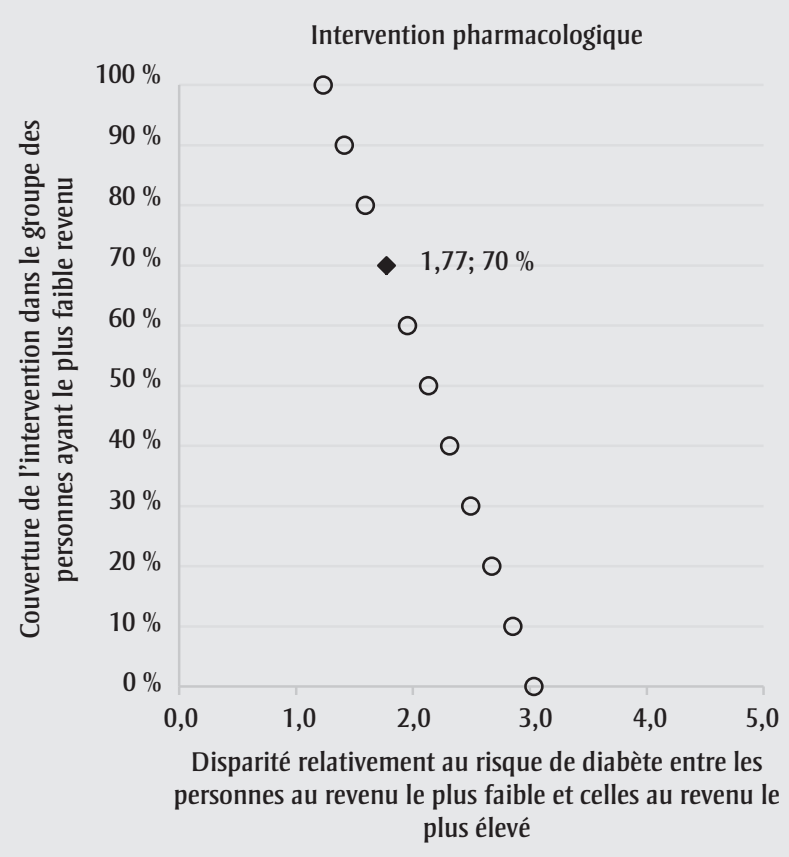

Intervention pharmacologique

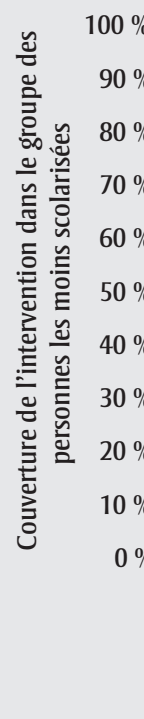

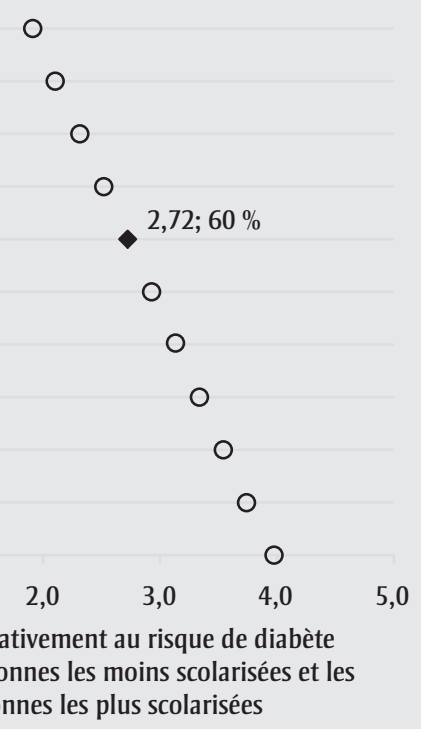

Remarque : Dans ces scénarios, on a présumé que la couverture était de $100 \%$ dans le groupe au statut socioéconomique le plus élevé et qu'elle était variable dans le groupe au statut socioéconomique le plus faible.

personnes du groupe au SSE le plus faible en même temps que la RAR. Nous avons aussi montré qu'une faible couverture peut exacerber la disparité de base en ce qui concerne le risque de diabète lors- qu'elle dépasse le point de bascule de l'équité. À ce seuil, la différence de couverture de l'intervention annule tout avantage sur le plan de l'équité conféré par cette intervention et entraîne une plus grande disparité dans le risque de diabète. Cherchant à expliquer cet effet prédit, d'autres ont formulé l'hypothèse que les interventions en santé pourraient renforcer les disparités lorsqu'il existe une plus 
forte probabilité que les groupes avantagés disposent de plus de ressources financières et de connaissances leur permettant de tirer profit des occasions d'améliorer leur santé que les groupes moins nantis, moins instruits et sans emploi ${ }^{13,23}$. Cependant, Anderson et collab. ${ }^{1}$ ont soulevé deux points pertinents : premièrement, plus la pente initiale du risque de base est prononcée, plus grand sera l'effet que la couverture sous-optimale devra exercer pour annuler la possibilité que les interventions en santé réduisent les disparités en santé ; deuxièmement, la couverture des interventions réagit aux mesures stratégiques et, étant donné la grande RAR associée à un SSE faible, les efforts peuvent être axés de manière productive sur l'amélioration de la couverture des interventions dans ce groupe de population afin de réduire les disparités.

\section{Points forts et limites}

Le recours à une enquête sur la santé de la population avec données autodéclarées pour définir la population à l'étude a fait en sorte que nos calculs de l'efficacité dans la collectivité comportaient à la fois des points forts et des limites. L'un des points forts est la capacité à calculer le risque de base de diabète avec tous les indicateurs importants du SSE à l'aide d'un algorithme permettant une analyse multivariée du risque en population générale. Ce type d'algorithme fait appel à des données personnelles tirées d'enquêtes sur la santé de la population et représente la méthode la plus discriminante pour estimer le risque de base ${ }^{6}$. L'utilisation d'une enquête sur la santé de la population a permis aussi une certaine flexibilité dans l'examen des différentes strates socioéconomiques et des populations cibles. De fait, nous avons pu faire varier individuellement, pour chaque répondant de l'enquête, la plupart des composantes de l'efficacité dans la collectivité, ce qui nous a permis de réaliser une vaste gamme d'analyses de la sensibilité. Nous avons fait varier l'efficacité des interventions en fonction de l'âge et de l'IMC, mais nous aurions aussi bien pu la faire varier en fonction d'autres caractéristiques. De même, nous avons fait varier la couverture en fonction des strates socioéconomiques, mais il aurait été facile de faire varier la couverture en fonction d'autres caractéristiques des répondants.

Nos résultats reposent sur l'hypothèse que le bénéfice relatif des interventions sur le mode de vie et des interventions pharmacologiques réalisées auprès des personnes à risque de diabète était constant quel que soit le SSE. À l'avenir, les applications du modèle que nous avons décrit seraient plus solides si l'on examinait l'effet d'une variation de l'efficacité des interventions en fonction du SSE. Toutefois, il existe certaines données probantes laissant croire que la prévention du diabète est d'une efficacité égale - en termes relatifs - dans toutes les strates socioéconomiques. À titre d'exemple, le Diabetes Prevention Program Research Group a fait état d'un bénéfice similaire en matière de prévention dans tous les groupes raciaux et ethniques faisant partie d'une étude réalisée dans 27 centres situés aux ÉtatsUnis $^{8}$. Les études examinées par Gillies et collab. ${ }^{7}$ ont été menées dans un large éventail de collectivités et chez un grand nombre de groupes ethniques dans le monde sans qu'une hétérogénéité n'ait été relevée. Par ailleurs, il a été établi que, en comparaison, les interventions sur le mode de vie étaient tout aussi efficaces dans les groupes à faible revenu, malgré une faible couverture ${ }^{24}$. Enfin, dans peu d'études et d'examens s'est-on penché sur la différence d'efficacité des interventions selon la strate socioéconomique. Pour combler cette importante lacune en matière de données, les Cochrane and Campbell Collaborations ont récemment préconisé une plus grande intégration de l'équité dans les examens et ont fourni un cadre pour faciliter le processus ${ }^{25}$.

\section{Conclusion}

L'efficacité d'une intervention préventive dans la collectivité peut se calculer à l'aide d'un modèle simple qui tient compte de la taille de la population cible, du risque de base de la maladie, de l'efficacité de l'intervention et de la couverture de l'intervention. La probabilité que les inter- ventions réduisent les inégalités peut être estimée lorsque l'efficacité dans la collectivité est calculée pour différentes strates socioéconomiques. Cependant, l'une des difficultés actuelles est l'absence de données sur le degré de couverture atteignable ou sur les différences probables dans les populations cibles et l'efficacité des interventions. Des analyses de sensibilité peuvent être réalisées pour estimer le point de bascule de l'équité, contexte dans lequel les effets de l'intervention passent de la réduction des inégalités à une augmentation paradoxale des disparités relativement au risque de maladie. Dans ces analyses de la sensibilité, on peut faire varier la couverture prévue ou d'autres composantes de l'efficacité dans la collectivité pour examiner leur influence plausible et renforcer les données probantes concernant la probabilité de réduire les inégalités en santé à l'aide de stratégies de prévention individuelles.

\section{Remerciements}

Nous remercions le $\mathrm{D}^{\mathrm{r}}$ Michael J. Pencina, de l'Université de Boston, d'avoir fourni la macro SAS permettant de calculer la calibration et la discrimination des modèles en survie afin de réaliser une analyse de validation (figurant dans l'annexe en ligne). Douglas G. Manuel est titulaire d'une chaire en santé publique appliquée des Instituts de recherche en santé du Canada et de l'Agence de la santé publique du Canada. John Lynch est titulaire d'une bourse de recherche australienne du National Health and Medical Research Council (NHMRC). L'étude a été financée par les Instituts de recherche en santé du Canada et le Réseau de recherche sur l'amélioration de la santé des populations du ministère de la Santé et des Soins de longue durée (MSSLDO) de l'Ontario. L'étude a été soutenue par l'Institut de recherche en services de santé (IRSS), lequel est financé par une subvention annuelle du MSSLDO. Les opinions, résultats et conclusions de l'étude n'engagent cependant que les auteurs et sont indépendants des sources de financement. Ils ne doivent pas être perçus comme étant approuvés par l'IRSS ou par le MSSLDO. 


\section{Références}

1. Anderson GM, Bronskill SE, Mustard CA, Culyer A, Alter DA, Manuel DG. Both clinical epidemiology and population health perspectives can define the role of health care in reducing health disparities. J Clin Epidemiol. 2005;58(8):757-62.

2. Furukawa TA, Guyatt GH, Griffith LE. Can we individualize the 'number needed to treat'? An empirical study of summary effect measures in meta-analyses. Int $\mathrm{J}$ Epidemiol. 2002;31(1):72-6.

3. McAlister FA. Commentary: relative treatment effects are consistent across the spectrum of underlying risks...usually. Int J Epidemiol. 2002;31(1):76-7.

4. Tugwell P, de Savigny D, Hawker G, Robinson V. Applying clinical epidemiological methods to health equity: the equity effectiveness loop. BMJ. 2006;332(7537): 358-61.

5. Galobardes B, Shaw M, Lawlor DA, Lynch JW, Davey Smith G. Indicators of socioeconomic position (part 1). J Epidemiol Community Health. 2006;60(1):7-12.

6. Manuel DG, Rosella LC. Commentary: assessing population (baseline) risk is a cornerstone of population health planning-looking forward to address new challenges. Int J Epidemiol. 2010;39(2):380-2.

7. Gillies CL, Abrams KR, Lambert PC et collab. Pharmacological and lifestyle interventions to prevent or delay type 2 diabetes in people with impaired glucose tolerance: systematic review and meta-analysis. BMJ. 2007;334(7588):299.

8. Knowler WC, Barrett-Connor E, Fowler SE et collab. Reduction in the incidence of type 2 diabetes with lifestyle intervention or metformin. N Engl J Med. 2002;346(6):393403.

9. Vermeire E, Wens J, Van Royen P, Biot Y, Hearnshaw $H$, Lindenmeyer A. Interventions for improving adherence to treatment recommendations in people with type 2 diabetes mellitus. Cochrane Database Syst Rev. 2005(2):CD003638.
10. Vermeire E, Hearnshaw H, Van Royen P, Denekens J. Patient adherence to treatment: three decades of research. A comprehensive review. J Clin Pharm Ther. 2001;26(5):33142.

11. Etches V, Frank J, Di Ruggiero E, Manuel D. Measuring population health: a review of indicators. Annu Rev Public Health. 2006; 27:29-55

12. Rosella LC, Manuel DG, Burchill C, Stukel TA. A population-based risk algorithm for the development of diabetes: development and validation of the Diabetes Population Risk Tool (DPoRT). J Epidemiol Community Health. 2011;65(7):613-20.

13. Frohlich KL, Potvin L. Transcending the known in public health practice: the inequality paradox: the population approach and vulnerable populations. Am J Public Health. 2008;98(2):216-21.

14. Capewell S, Graham H. Will cardiovascular disease prevention widen health inequalities? PLoS Med. 2010;7(8):e1000320.

15. Rose G. Environmental health: problems and prospects. J R Coll Physicians Lond. 1991;25(1):48-52.

16. McLaren L, McIntyre L, Kirkpatrick S. Rose's population strategy of prevention need not increase social inequalities in health. Int J Epidemiol. 2010;39(2):372-77.

17. O’Brien BJ, Heyland D, Richardson WS, Levine M, Drummond MF. Users' guides to the medical literature. XIII. How to use an article on economic analysis of clinical practice. B. What are the results and will they help me in caring for my patients? Evidence-Based Medicine Working Group. JAMA. 1997;277(22):1802-6.

18. Drummond MF, Chaperon, J, Carrère, M-O. Méthodes d'évaluation économique des programmes de santé. Paris : Économica; 1998.

19. Ackermann RT, Finch EA, Brizendine E, Zhou H, Marrero DG. Translating the Diabetes Prevention Program into the community. The DEPLOY Pilot Study. Am J Prev Med. 2008;35(4):357-63.

20. Anderson RM, May RM. Directly transmitted infectious diseases: control by vaccination. Science. 1982;215:1053-60.
21. Wamala S, Merlo J, Bostrom G, Hogstedt C, Agren G. Socioeconomic disadvantage and primary non-adherence with medication in Sweden. Int $\mathrm{J}$ Qual Health Care. 2007;19(3):134-40.

22. Organisation mondiale de la santé. Adherence to long-term therapies: evidence for action. Genève $(\mathrm{CH})$ : Organisation mondiale de la santé; 2003.

23. Mechanic D. Disadvantage, inequality, and social policy. Health Aff. (Millwood). 2002; 21(2):48-59.

24. Jackson R, Lawes CM, Bennett DA, Milne RJ, Rodgers A. Treatment with drugs to lower blood pressure and blood cholesterol based on an individual's absolute cardiovascular risk. Lancet. 2005;365(9457):43441.

25. Tugwell P, Petticrew M, Kristjansson E et collab. Assessing equity in systematic reviews: realising the recommendations of the Commission on Social Determinants of Health. BMJ. 2010;341:c4739. 